\title{
En la ruta de la acreditación institucional de alta calidad
}

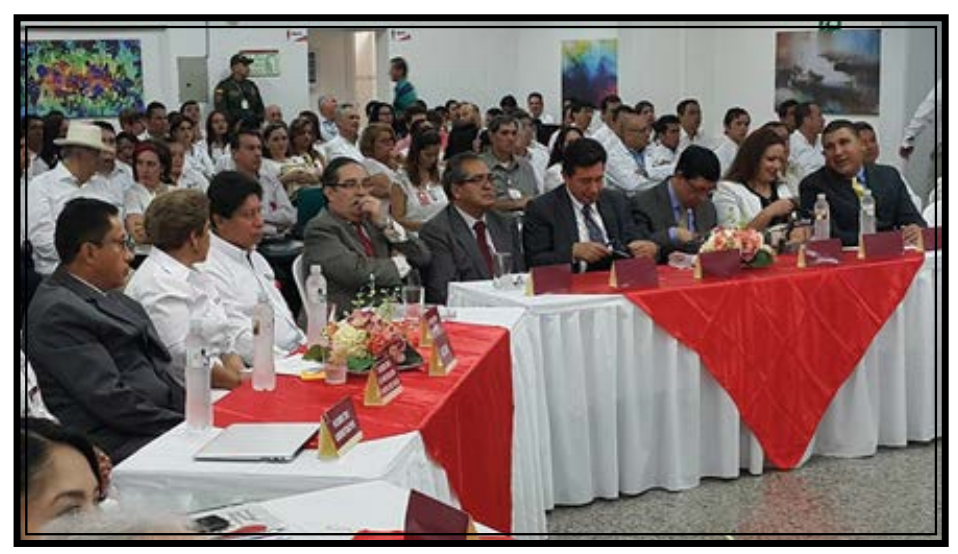

El Ministerio de Educación Nacional (MEN) cuenta con el sistema Nacional de Acreditación cuyo objetivo es "Garantizar a la sociedad que las instituciones de Educación Superior, que hacen parte del Sistema, cumplan con los más altos requisitos de calidad y realicen sus propósitos y objetivos". Este sistema se apoya en el Consejo Nacional de Acreditación (CNA), instancia que preside y organiza el proceso de acreditación, apoyándose en los documentos de lineamientos, criterios e indicadores para la autoevaluación de programas (pregrado y posgrado) así como de los procesos institucionales.

Con este referente, la Universidad Surcolombiana asume el compromiso con la calidad como el principio que orienta de manera permanente el Proyecto Educativo Universitario (PEU) y que permea todas las acciones desarrolladas en el marco del Plan de Desarrollo Institucional (PDI 2015-2024). Es así, como en febrero del 2017, la Institución presentó ante el C.N.A el informe de autoevaluación con fines de la Acreditación Institucional.

Con esta orientación, en los últimos años, la Institución ha vivido un proceso de modernización de sus estructuras académicas y ha definido un plan de desarrollo de largo aliento que le permiten consolidarse como una Universidad de docencia que hace investigación, para mantener y acrecentar su pertinencia social, académica y científica, sin perder la riqueza acumulada por la tradición y las notas características que han diferenciado al Surcolombiano a lo largo de su existencia.

Para ello, la preocupación constante por asegurar la calidad de las actividades académicas y administrativas le ha permitido fortalecer la cultura de la autoevaluación y la autorregulación permanente, así como la puesta en marcha de modelos y mecanismos institucionales orientados a facilitar los procesos de evaluación, mejoramiento continuo y certificación de la calidad.

Para el logro de las metas planteadas, la Universidad establece el proceso de autoevaluación con miras a la acreditación de programas académicos e institucional. Como resultado de este direccionamiento estratégico, la Institución cumplió con la apreciación de condiciones iniciales en el año 2013 y la respectiva visita de verificación de ellas se realizó en octubre de 2014 y el acto administrativo de aval que permitió el inicio del proceso de autoevaluación en marzo de 2015, con el propósito fundamental de realizar un proceso participativo, por medio del cual la Institución, por sí misma, identificara, obtuviera y analizara información útil, válida y confiable acerca de sus fortalezas y debilidades, de las amenazas y oportunidades del entorno, encaminada a juzgar 
alternativas de decisión para lograr efectividad $\mathrm{y}$ eficiencia en sus procesos de planeación y cambio para el desarrollo institucional. Dicho ejercicio de autoevaluación fue desarrollado bajo un enfoque sistémico, en el cual cada uno de los componentes internos de la Universidad fue considerado como un organismo con dinámica propia que interactúa con los otros.

En el mes de diciembre de 2016, concluyó el proceso con un informe general, en el que se consignaron los objetivos, metodologías y las principales recomendaciones en lo académico y en lo administrativo. La implementación de las acciones propias del Plan de Desarrollo Institucional y del plan de mejoramiento -resultante de la autoevaluación- es asumido por la Dirección de la Institución, lo que dio inicio a un período de transformación, considerando que la Institución se ve de manera permanente obligada a realizar una serie de cambios, unos más profundos que otros, para responder de manera adecuada a la tarea que le impone no sólo su objeto social, sino -y si se quiere con mayor intensidad- el entorno social al cual se debe, los cambios tecnológicos cada día más exigentes y las modificaciones en la manera como la sociedad percibe el papel de sus instituciones. Para la Universidad esta obligación responde no sólo a la necesidad de adecuarse, sino que está implícita en su razón de ser, que la obliga a repensarse permanentemente, a mantener un espíritu de autocrítica, a recibir y discutir toda hipótesis, en fin, a convertirse en la conciencia crítica de la sociedad.

En síntesis, entre 2015 y 2016, la Universidad Surcolombiana, se sometió a un proceso de autoevaluación multidimensional, caracterizado por la apertura de espacios de participación para toda la comunidad universitaria y por la coordinación colectiva, pues todas las actividades fueron sometidas a consideración del Comité de Acreditación Institucional, en el cual toman asiento los decanos o sus delegados, representantes de estudiantes y delegados de instancias administrativas-directivas (ORNI, Proyección Social, Bienestar Universitario, División Financiera, CTIC y otros); y del Comité Administrativo integrado por los directores de Talento y Desarrollo Humano, de Control Interno, Gestión de Calidad, Oficina de Planeación, Secretaria General, Vicerrectores Académico, Investigación y Administrativo y el Rector.

La autoevaluación, como parte integral del quehacer académico universitario, parte del supuesto de que la acción universitaria se da en un marco de calidad; esto es, dentro de una búsqueda permanente de la excelencia que implicó una metodología centrada en el análisis de procesos para examinar la dinámica de la Institución, teniendo como punto de partida el hecho de que ésta, se crea para llevar a cabo ciertos propósitos $\mathrm{u}$ objetivos perdurables, mediante la ejecución de una secuencia articulada de actividades.

De esta manera, el análisis de procesos ha permitido alcanzar una visión sistémica de la Institución como el transcurrir continuo de un conjunto articulado de procesos, subprocesos y actividades, uno de los cuales toma el carácter de dominante y los otros de subordinados. En tal sentido, el análisis de procesos hace énfasis en el desarrollo de la Misión Institucional, razón de ser de la Institución, y en todo lo que le es pertinente; se busca la coherencia entre lo que se enuncia, lo que se hace y lo que se obtiene (los resultados). Dado que se trata de una acción colectiva, pues la misión se lleva a cabo mediante la cristalización de múltiples procesos, subprocesos y actividades, cuya responsabilidad recae en las unidades organizacionales y en los equipos de trabajo especializados e interdisciplinarios, y no en los individuos.

Dicho análisis facilitó la autoevaluación institucional de carácter integral, en donde la Universidad se examinó como un todo complejo articulado que tiene unos objetivos de corto, mediano y largo plazo, integrados de manera sistémica, alrededor de la Visión y Misión Institucionales.

De acuerdo con las reflexiones anteriores, ha sido factible, conveniente y adecuado, el adoptar un modelo de autoevaluación institucional, para la Universidad Surcolombiana, fundado 
en el análisis de un sistema de procesos y subprocesos, que emanan del compromiso social establecido en la Misión. Este sistema tiene tres componentes principales, ordenados de acuerdo con su trascendencia en la Misión: a) están los procesos esenciales o que dan vida a la misión institucional, los cuales se relacionan directamente con los programas de formación (pregrado y postgrado), con las actividades de investigación y con las de extensión; b) están los procesos de apoyo, tanto académico como administrativo; y c) se identifican los procesos de dirección, responsables de trazar el rumbo de la Institución. Esta manera de abordar la autoevaluación institucional, dio lugar a la conformación de tres mesas de trabajo lideradas cada una por las Vicerrectorías que integraron los diferentes factores contemplados en el Modelo de Autoevaluación del CNA:

- Mesa 1, Vicerrectoría Académica. Factores: Misión y Proyecto educativo, Estudiantes, Profesores y procesos académicos.

- Mesa 2, Vicerrectoría de Investigaciones y Proyección Social. Factores: Visibilidad Nacional e Internacional, Investigación y Creación Artística y Pertinencia e Impacto Social.

- Mesa 3, Vicerrectoría Administrativa. Factores: Bienestar Institucional; Organización, gestión y administración; Recurso de Apoyo Académico e Infraestructura y Recursos Financieros.

En síntesis, la autoevaluación por procesos ha dado respuesta a todos y cada uno de los factores con los cuales se evalúa la calidad en las instituciones de educación superior. Además, ha traído dos beneficios importantes: en primer lugar, genera una visión sistémica y sistemática de las actividades institucionales, las cuales se convierten en responsabilidades colectivas, de equipos de personas y no de individuos aislados. En segundo lugar, estimula el fortalecimiento de una cultura de autocontrol y de mejoramiento continuo, pues permite prevenir los errores y reducir los costos derivados de la adopción de medidas correctivas.

El análisis de procesos en la implementación de la autoevaluación institucional orientada a la obtención de la acreditación de la Universidad, además de la incorporación en el PDI de los planes de mejoramiento y del seguimiento a los proyectos de fortalecimiento, consolidación e innovación - definidos a partir de los diversos procesos de evaluación-, se realizaron las diferentes acciones definidas en el modelo de Autoevaluación y Autorregulación de la Universidad, que involucraron a toda la comunidad académica de la Institución, en la búsqueda de una valoración objetiva y transparente. Estas fueron:

I. Fase de contextualización: La reflexión que la Universidad Surcolombiana adelanta sobre su ser, su quehacer y su deber ser, no es una acción reciente, es un proceso que se ha venido desarrollando de manera sistemática en los últimos tiempos. Lo que la Universidad ha entendido por "cultura de la autoevaluación" y los parámetros que han orientado el diseño y construcción del Plan de Desarrollo, así como los elementos que se han tenido en cuenta para avanzar en el proceso de autoevaluación y autorregulación, son las bases conceptuales y prácticas sobre las cuales reposa la reflexión en torno al quehacer histórico de la Institución y de sus Programas.

Los procesos de autoevaluación y autorregulación, son dinámicos y permanentes, incluyen el seguimiento de los estudios y evaluaciones que se han desarrollado como parte del proceso, ya sean formales o informales, ya que ellos constituyen la base para la identificación de la calidad de los procesos adelantados como resultado de los ejercicios de autoevaluación. Además, ha permitido identificar las acciones que se integraron al PDI y confrontar los resultados de los proyectos formulados.

\section{Fase conceptual: Conllevó a:}

a. Revisión y Ajuste del modelo de autoevaluación, integrando las definiciones institucionales sobre mecanismos e instancias de autorregulación y afinando los procedimientos institucionales para tal fin.

b. Valoración de factores y características, entendida como la incidencia de los distintos factores y características que integran el modelo de evaluación del CNA, expresando así la 
importancia de cada elemento respecto de los demás, para asegurar la calidad en el logro del Proyecto Educativo Universitario (PEU), en el cumplimiento de la Misión de la Universidad. En esta actividad, además del Comité Institucional de Autoevaluación y Acreditación, se contó con la participación de directivos de diferentes unidades y con representantes de profesores, estudiantes y egresados de la Universidad.

III. Fase metodológica instrumental: Esta fase permitió diseñar guías para la solicitud de documentos e información numérica y codificación de registros y archivos de información. Matrices como la Matriz general de factores, características y fuentes. Además, tablas, indicadores cuantitativos, Instrumentos para encuestas a profesores, estudiantes, egresados, administrativos y directivos, así como entrevistas a directivos y comunidad externa.

IV. Fase operativa de campo: Es la fase que puso en acción las actividades de autoevaluación propiamente dichas. a. Recolección de toda la Información de la Universidad: La información de tipo documental, numérica y estadística, se recolectó tanto desde el sitio Web, como desde la Intranet de la Universidad; así mismo se solicitaron documentos específicos a las diferentes dependencias y la elaboración de informes detallados, necesarios para el análisis descriptivo de la institución (Se elaboraron 25 solicitudes de documentos y tablas para todas las dependencias de la Universidad, 129 documentos y 50 tablas). De igual manera, la información numérica y estadística se recopiló usando sus bases de datos y algunos sistemas de información como los boletines estadísticos institucionales.

b. Para la recolección de la información relacionada con la percepción y valoración de la calidad, por parte de la comunidad universitaria, en donde participaron estudiantes, profesores, administrativos y directivos, a quienes se les aplicaron encuestas; las entrevistas fueron dirigidas a la comunidad externa. Y finalmente se desarrollaron talleres y grupos focales con los segmentos específicos de la población (estudiantes y profesores).
V. Fase descriptiva y valorativa: Partiendo de la información obtenida, se realizó el análisis cualitativo y cuantitativo que permitió dar cuenta de los avances alcanzados por la Universidad en los últimos cinco años. Posterior a esto se definieron grupos de trabajo para la valoración donde se incluyeron representantes de todos los estamentos de la Universidad; estas personas previa lectura del primer documento borrador asistieron a los talleres programados con el fin de analizar la información recopilada, emitir juicios valorativos tendientes a dejar en claro qué tan cerca o distante se encuentra cada una de las características del ideal y qué avances y mejoras se han dado a través del tiempo y así de esta manera, calificar el cumplimiento de cada característica, de acuerdo con una escala definida.

De esta manera, durante el proceso de autoevaluación, se realizaron 30 talleres de valoración, en los que participaron 802 representantes de la comunidad institucional (directivos, administrativos, profesores, estudiantes y egresados). Y además, se realizaron siete grupos focales adicionales de valoración con más de 100 estudiantes de las 7 facultades. Una vez finalizado lo anterior, se dio paso a la elaboración del documento final de evaluación institucional, que concluyó con la presentación de la valoración global de la institución a manera de conclusiones, además de la identificación de fortalezas y debilidades y el planteamiento de temas de mejoramiento.

\section{Valoración global de la calidad de la institución}

Es posible concluir que la Universidad Surcolombiana en los últimos años ha fortalecido su capacidad de gestión para responder a los retos impuestos en su Misión, su Visión, su PEU y su PDI y en concordancia con las actuales tendencias mundiales en educación superior.

La calidad y la pertinencia como criterios que guían su actuación, han permitido que en el periodo comprendido entre el 2012 y el 2017 se hayan fortalecido las capacidades institucionales de planeación estratégica, evaluación y 
mejoramiento permanente y se haya arraigado la cultura de la autoevaluación y la autorregulación en los miembros de la comunidad Universitaria.

En la autoevaluación finalizada en el 2016 se evidencian los logros de la Universidad en los diferentes aspectos que componen el modelo del CNA; igualmente aparecen debilidades que la Institución debe seguir abordando para lograr, en el tiempo, acercarse a sus ideales de calidad y continuar respondiendo a la sociedad con compromiso y responsabilidad.

Este documento fue presentado al C.N.A el 9 de febrero de 2017. Esta actividad arrojo resultados cuantitativos, otorgando la calificación global para la universidad de 4.48 .

VI. Visita de pares externos: Los Pares Externos son Expertos Académicos, designados por el CNA, quienesjuzgaron cómo ha sido la evolución de todos los procesos de la Universidad desde su decisión de obtener la acreditación (2011), a partir de referentes universales y particulares de la educación superior, de la Institución y desde el modelo CNA.

La comisión de pares designados por el CNA, conformada por profesionales de prestigiosas universidades nacionales y extranjeras y con formación idónea para llevar a cabo esta actividad, realizaron la visita de autoevaluación durante los días 7, 8 y 9 de junio, para lo cual la Institución con sus equipos responsables puso a su disposición todos los documentos importantes para su evaluación, así como aquellos que se consideraron pertinentes y fueron solicitados. De otra parte, se aseguró la disponibilidad de la comunidad académica-administrativa (directivos, profesores, estudiantes, egresados y personal administrativo), con quienes los pares se entrevistaron. Esto permitió visualizar la importancia de la participación de los miembros de la comunidad académica para cada una de las reuniones a las que fueron convocados; por ello, previamente se les contextualizó en forma clara sobre cada uno de los aspectos que se vienen desarrollando en la Universidad, y así dieron cuenta de la evolución de la misma, bajo una perspectiva real, objetiva y transparente.
Posterior a la visita de pares externos, estos elaboraron un informe que presentaron al CNA, instancia que lo envió a la Universidad. Este informe, expresa que la Universidad Surcolombiana ha realizado esfuerzos inclinados a mejorar y transformar la calidad integral de la Institución, al indicar que la Universidad obtuvo una Valoración positiva: seis factores con valoración de "Cumple en Alto Grado" (Procesos Académicos; Visibilidad nacional e internacional; Investigación y creación artística; autoevaluación y autorregulación; Organización, Gestión y Administración y Recursos de Apoyo Académico e Infraestructura. Y, seis factores con valoración de "Cumple Plenamente" (Misión y Proyecto Institucional; Estudiantes, Profesores, Pertinencia e impacto social; Bienestar Institucional y Recursos Financieros).

Al valorar el informe en relación al proyecto institucional $\mathrm{y}$ en general al que hacer universitario, se identifican 45 fortalezas, 11 debilidades, 18 sugerencias, 1 juicio de valor y 17 recomendaciones. Destaca las acciones emprendidas por la Universidad Surcolombiana, conjuntamente con la Gobernación, representantes y senadores del Departamento del Huila, que dio lugar a la Ley de Estampilla USCO que permitirá a la Institución inversiones por más de 300 mil millones en la próxima década; además que la Institución cuenta con tres certificaciones de calidad relacionadas con: la norma ISO 9001:2008, desde el año 2011, proceso que fue renovado en el año 2017 y que contemplo el 100\% de sus procesos; NTCGP 1000:2009 de fecha enero del año 2017 y la certificación internacional de alta calidad IQNet desde el año 2011 que indica que sus procesos administrativos cumplen normas internacionalmente aceptadas de calidad.

No obstante, hace énfasis especial en todos y cada uno de los factores con fortalezas, aspectos a mejorar y recomendaciones. La institución ha integrado los 12 factores en tres grandes mesas, Formación, Investigación y Administración.

La Mesa No. 1, integrada por los Factores de Misión y Proyecto Institucional, Estudiantes, Profesores y Procesos Académicos, se identifican 
enelinformedepares 16Fortalezas, 11 debilidades y/o sugerencias, 76 recomendaciones, en donde se destacan las siguientes:

"Los pares apreciamos de las reuniones sostenidas con los directivos, administrativos, egresados, empleadores estudiantes y docentes que la Universidad impregna a la comunidad académica y administrativa con los postulados de la misión - visión y que las mismas se vivencian de una manera que impacta el proyecto académico, la formación integral y constituye un sello institucional."

"Sistema de estímulos, descuentos y otros servicios de apoyo para estudiantes basados en el desempeño académico, por participación en actividades deportivas, culturales, artísticas y monitorias"

"Su planta profesoral ha mostrado una mejora considerable en los últimos años reflejado en los indicadores relativos a sus calificaciones académicas y en los buenos porcentajes de doctores y magíster"

"Al estar recientemente propuesta la misión y su PEU falta la interiorización general de sus postulados en el cuerpo estudiantil"

"Ampliar y reforzar el desarrollo de competencias en lengua extranjera"

“Continuar y profundizar los programas $y$ servicios de apoyo para promover la permanencia y graduación de los estudiantes, como mecanismo para disminuir la tasa de deserción estudiantil"

"La Comisión aprovecha la oportunidad para animar a la Universidad a seguir con la implementación de su PEU, con particular énfasis en encontrar los mecanismos idóneos para implementar el proyecto desarrollo de la institución en lo que respecta al fortalecimiento del trabajo en equipo y de la construcción de una comunidad académica cada vez más comprometida con el desarrollo de su comunidad"
De la Mesa 2, integrada por los factores de Investigación, Proyección Social, Internacionalización, se destacan 11 Fortalezas, 12 debilidades y/o sugerencias y 9 recomendaciones, se destacan:

"Un importante número de convenios interinstitucionales que reflejan la interacción de la Universidad con instituciones locales y departamentales, así como con instituciones académicas del ámbito internacional"

“Cualificación de la actividad investigativa, existencia de grupos y proyectos que inciden en la proyección social de la Institución"

"La fuerte presencia e influencia en el ámbito local en los diferentes sectores económicos $y$ sociales de la ciudad que hacen honor a su nombre"

"Es necesario incrementar la visibilidad de sus investigadores y respaldar las colaboraciones internacionales"

"La VIPS debe continuar con sus esfuerzos a fin de incrementar la participación de todos los profesores en actividades de investigación, reforzando los grupos de investigación existentes (y no necesariamente con la creación de nuevos grupos) mejorando su categorización en el sistema nacional de CTI"

"Seguir fortaleciendo la proyección de la Universidad en su entorno y su aporte a dinamizar procesos de innovación, esto incluye fortalecer la formación en emprendimiento y el apoyo a la aplicación de los resultados de la investigación en las empresas y el sector productivo del país"

"Promover de manera más frecuente la internacionalización de los programas de formación profesional, así como de los programas y proyectos de investigación"

"Promocionar la imagen de la Universidad en el exterior del país, a través de los medios de comunicación e información disponibles $u$ otros que sean apropiados" 
La Mesa 3, conformada por los Factores de Bienestar Universitario, Gestión y Administración, Recursos de Apoyo Académico e Infraestructura y Recursos financieros, plantea en el Informe de Pares, 15 Fortalezas, 5 debilidades o sugerencias y 1 recomendación, las cuales se expresa así:

"Los miembros de la comunidad universitaria muestran un alto grado de satisfacción sobre su Bienestar Universitario, por ser incluyente con todos sus estamentos, la diversidad y calidad de los servicios $y$, sobre todo, por permitir el desarrollo integral individual y mejorar la convivencia institucional."

"Clara y positiva diferenciación institucional que se proyecta al futuro gracias a la existencia de un plan de desarrollo que propone una visión y que cuenta con los dispositivos necesarios para hacer su seguimiento"

"Se destaca igualmente, la politica de inversión en recursos de planta física y de apoyo académico"

"La Universidad cuenta con una sólida estructura financiera caracterizada por una identificación precisa de sus fuentes"

"No existen herramientas adecuadas que permitan la evaluación periódica e impacto de los programas y actividades que ofrece Bienestar Universitario"

"Definir y enunciar indicadores de desempeño sobre gestión de la proyección de la Universidad en los ámbitos nacional e internacional"

"El uso de los recursos tecnológicos de apoyo académico no está suficientemente extendido y aprovechado"

"Continuar con los esfuerzos de diversificación de las fuentes de financiamiento para garantizar el desarrollo sostenible de la universidad y mantener proactivamente la correspondencia entre la planeación, el presupuesto y ejecución de los recursos disponibles en correspondencia con las necesidades del desarrollo académico de la institución"

Una vez recibido el Informe de Pares, la Institución contó con 15 días calendario para radicar ante el CNA un informe de Respuestas y/o comentarios. Es así, como la Oficina de Acreditación, junto con los líderes de cada Mesa e integrantes de cada factor y el Comité de Acreditación Institucional, revisaron dicho Informe y generaron los argumentos pertinentes; ejercicio que luego de varias reuniones, se complementó con Jefes de Oficinas y Comité Directivo, quienes valoraron el informe de una manera reflexiva y analítica, ponderando de manera positiva la actitud y responsabilidad objetiva con que los Pares Externos asumieron su rol, y acatando las fortalezas, debilidades y recomendaciones expresadas en el Informe. El documento de respuesta, "Comentarios del Rector al Informe de Pares Externos" fue enviado por correo electrónico el 31 de agosto, y radicado en el CNA el 1 de septiembre.

Este documento de 39 hojas, contiene unas consideraciones generales, las consideraciones a cada uno de los juicios del informe sobre los cuales había lugar a argumentación o aclaración, la gradación en los juicios sobre la calidad alcanzada por la Institución, comentarios a las principales debilidades y unas recomendaciones para el mejoramiento de la calidad institucional.

VII. Fase autorreguladora: A partir de las valoraciones de cada factor donde se identificaron los temas de mejoramiento $y$ de desarrollo, se clasificaron para identificar los proyectos de mejoramiento y los de consolidaciones resultantes de la autoevaluación 2016. Es de tener en cuenta que, algunos proyectos ya se vienen desarrollando en la Universidad y, en este caso, se ha realizado su revisión con la finalidad de afinar sus objetivos, metas y alcances. Igualmente, estos también se incorporan al PDI de la Universidad, asignando tiempos y responsables para su cumplimiento. Se continúa avanzando en los proyectos que permitan alcanzar los retos planteados como resultado de los procesos de autoevaluación 
y se definieron nuevos retos que redundarán en la calidad de la formación impartida por la Universidad.

En la actualidad, la Institución se encuentra en la elaboración, implementación y seguimiento de Proyectos de Mejoramiento y Consolidación como base para la aproximación a los procesos de mejoramiento y autorregulación del informe presentado al CNA, a partir del análisis que permite realizar el cruce de aspectos relevantes y de precisar las fortalezas y debilidades comunes y significativas para el crecimiento de la Institución, lo cual ha sido base para la definición de proyectos.

Este ejercicio es vital como preparación para la autoevaluación con fines de renovación de la acreditación, pues permite, por una parte, recopilar organizadamente las acciones ejecutadas y plasmarlas en forma de proyectos; $y$ por otra, proyectar nuevas acciones inmediatas, a corto plazo y a largo plazo. Todo lo anterior servirá como información clave que dará cuenta del proceso de autorregulación institucional.

Finalmente, el CNA realiza el correspondiente análisis, para posteriormente enviar al Ministerio de Educación Nacional la recomendación de otorgar la ACREDITACIÓN A LA UNIVERSIDAD SURCOLOMBIANA. Los procesos que se adelantan en la Universidad redundan en beneficios para toda la comunidad, así mismo requieren de la participación de todos para el logro de las metas planteadas. La calidad es responsabilidad de todos, infórmate y participa, así ganamos todos.

\section{Unidad Aseguramiento de la Calidad Universidad Surcolombiana}

